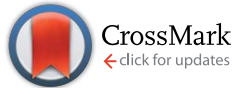

Cite this: RSC Adv., 2017, 7, 9948

Received 28th November 2016 Accepted 25th January 2017

DOI: $10.1039 / c 6 r a 27462 e$

rsc.li/rsc-advances

\section{Highly efficient visible-light-driven plasmonic photocatalysts based on graphene oxide mediated hybridization of graphite and $\mathrm{Ag} / \mathrm{AgBr} \dagger$}

\author{
Guangwei Geng, ${ }^{\text {ab }}$ Bo Guan, ${ }^{b}$ Penglei Chen, ${ }^{\star a b c}$ Mingshan Zhu, ${ }^{b}$ Changchun Yang ${ }^{a}$ \\ and Minghua Liu bc
}

Graphite (Gr)-based composite materials have been attracting great interest from the scientific and engineering communities. While tremendous progress has been achieved in this field, it still remains a formidable challenge to hybridize pristine $\mathrm{Gr}$ with other components under ambient conditions without breaking the $\pi$-conjugated system of Gr. Herein, we report that Gr could be facilely hybridized with visible-light-driven plasmonic photocatalyst $\mathrm{Ag} / \mathrm{AgBr}$ with the assistance of graphene oxide (GO) nanosheets under ambient conditions. We demonstrate that without the use of GO, Gr could not be well hybridized with $\mathrm{Ag} / \mathrm{AgBr}$, while a good hybrid $\mathrm{Gr} / \mathrm{GO} / \mathrm{Ag} / \mathrm{AgBr}$ could be produced when $\mathrm{GO}$ nanosheets are introduced into the system. Our results show that compared to $\mathrm{Ag} / \mathrm{AgBr}, \mathrm{GO} / \mathrm{Ag} / \mathrm{AgBr}$ and $\mathrm{Gr} / \mathrm{Ag} /$ $\mathrm{AgBr}$ species, thus-fabricated $\mathrm{Gr} / \mathrm{GO} / \mathrm{Ag} / \mathrm{AgBr}$ hybrids could display substantially enhanced catalytic activity towards the photobleaching of methyl orange under visible-light irradiation. It is disclosed that the good hybridization between $\mathrm{Gr}$ and $\mathrm{Ag} / \mathrm{AgBr}$, which is mediated by $\mathrm{GO}$, and the facilitated separation of photogenerated electrons and holes, which is promoted both by GO and Gr, contribute much to the boosted catalytic performances. Considering the excellent hybridization capability of GO, the exceptional electronic characteristics, versatile multifunctionality and low-cost of Gr, our protocol might be applicable not only to the $\mathrm{Ag} / \mathrm{AgBr}$-based plasmonic photocatalysts but also to a wide variety of other functional materials, wherein $\mathrm{Gr} / \mathrm{GO}$-based advanced materials of desired and reinforced functions or properties might be inaugurated.

\section{Introduction}

Graphene, which refers to a single-atom-thick two-dimensional $\mathrm{sp}^{2}$-bonded carbon atoms that are arranged in a hexagonal honeycomb lattice, has aroused numerous expectations. ${ }^{\mathbf{1 - 2 1}}$ This stems from its unique electrical, mechanical and physicochemical attributes, which endow it with promising prospects in various areas of paramount importance. ${ }^{\mathbf{1 - 1 7}}$ In the frontier field of graphene engineering, the hybridization of pristine graphene with other components is recognized to be one of the most important avenues to realize or to reinforce their function. ${ }^{\mathbf{1 - 7}}$ The perfect $\pi$-conjugated system of pristine graphene helps it preserve its unique attributes, scientifically

${ }^{a}$ College of Chemistry and Molecular Engineering, Zhengzhou University, 100 Science Road, Zhengzhou 450001, Henan, China

${ }^{b}$ Beijing National Laboratory for Molecular Science, Institute of Chemistry, Chinese Academy of Sciences, No. 2 Zhongguancun Beiyijie, Beijing 100190, China. E-mail: chenpl@iccas.ac.cn

'University of Chinese Academy of Sciences, Beijing 100049, China

$\dagger$ Electronic supplementary information (ESI) available: The SEM images of our $\mathrm{Ag} / \mathrm{AgBr}$ and $\mathrm{GO} / \mathrm{Ag} / \mathrm{AgBr}$ structures. The EDX elemental analysis and XPS spectra of our $\mathrm{Ag} / \mathrm{AgBr}$ and $\mathrm{Gr} / \mathrm{GO} / \mathrm{Ag} / \mathrm{AgBr}$ structures. See DOI: 10.1039/c6ra27462e facilitating an investigation on the intrinsic nature of graphene, and technically favoring the construction of advanced hybrids of reinforced performances. ${ }^{1-7}$ Nevertheless, there generally exist two limitations with regard to this issue, which to some extent disfavor the development of pristine graphene-based hybrids. First, from the viewpoint of availability, pristine graphene is commonly produced via mechanical exfoliation, chemical vapor deposition, etc. ${ }^{18-21}$ Either the lack of an accurate reproducibility or the requirement of a careful control on the experimental parameters limits a scalable production. ${ }^{18-21}$ Second, from the viewpoint of hybridization, the lack of hybridizable sites on the skeleton of pristine graphene and its poor solution processability make it difficult to realize a facile and bulk hybridization under ambient conditions. ${ }^{1-7}$

Practically, graphene oxide (GO) has gained much attention as a promising alternative of the pristine graphene. ${ }^{8-17}$ Structurally, GO is a polyaromatic fragment of pristine graphene with its skeleton being decorated with various oxygen-containing groups. Thanks to its scalable availability, good solution processability and the existence of fertile hybridizable sites on its skeleton, GO-based hybrids actually represent one of the main achievements of carbonaceous material engineering..$^{\mathbf{8 - 1 7}}$ However, the presence of oxygen-containing groups and defects 
on GO's skeleton destroy its $\pi$-conjugated system. This leads to a decrease in its electrical characteristics, and is generally undesired from the viewpoint of materials' performances. ${ }^{8-15,22,23}$ To overcome this, reduced GO (RGO), whose $\pi$-conjugated system is repaired to some extent, has been used to construct graphene-based hybrids, where enhanced performances could be realized..$^{\mathbf{8}-15,22,23}$ In most of the cases, various chemicals, electrolytes, dangerous gases, or high temperature are commonly required to obtain $\mathrm{RGO},{ }^{22-25}$ which in turn makes the manufacturing of RGO-based hybrids a relatively tedious work. Accordingly, a facile hybridization of these carbonaceous materials simultaneously characterized with easy availability, good $\pi$-conjugated system, and abundant hybridizable sites is a significant subject deserving to be explored intensively.

Among various carbonaceous materials, graphite (Gr), which consists of coherently stacked layers of pristine graphene, is among the most low-cost carbon sources. ${ }^{26,27}$ Its perfect $\pi$-conjugated system confers it with an electron mobility of as high as ca. $1.2 \times 10^{4} \mathrm{~cm}^{2} \mathrm{~V}^{-1} \mathrm{~s}^{-1} \cdot{ }^{28}$ Similar as the case of pristine graphene, the lack of hybridizable sites on Gr's skeleton and its poor solution processability, however, limit its hybridization under ambient conditions, ${ }^{26,27}$ although it is strongly desired to make a full use of its $\pi$-conjugated system in the hybrids. Recently, it has been reported that driven by the $\pi-\pi$ interactions between the $\pi$-conjugated aromatic domains of GO and the $\pi$ system of Gr, powdery Gr could be easily dispersed in aqueous dispersion of GO under ambient conditions. ${ }^{29,30}$ This might provide researchers with great opportunities to fabricate Gr-based hybrids of good $\pi$-conjugated system via an introduction of GO nanosheets into the system.

Recently, silver/silver halide-based species (Ag/AgX, X $=\mathrm{Cl}, \mathrm{Br}$, I) have drawn great attention as promising plasmonic photocatalysts towards the photobleaching of organic pollutants. ${ }^{31-47}$ This is owing to their exceptional visible-light-responsive photocatalytic performances, although silver halides conventionally function as the primary source material in photographic plates and are unstable upon photoirradiation. Inspired by the aboveaddressed issues, we herein report that Gr, which originally has a poor hybridization capability, could be easily yet well hybridized with the $\mathrm{Ag} / \mathrm{Ag} \mathrm{Br}$ plasmonic photocatalysts in the presence of GO nanosheets under ambient conditions. Compared to $\mathrm{Ag} / \mathrm{AgBr}$, the hybrids of $\mathrm{GO}$ and $\mathrm{Ag} / \mathrm{AgBr}(\mathrm{GO} / \mathrm{Ag} / \mathrm{AgBr}), \mathrm{Gr}$ and $\mathrm{Ag} / \mathrm{AgBr}(\mathrm{Gr} / \mathrm{Ag} /$ $\mathrm{AgBr}$ ), the as-fabricated $\mathrm{Gr} / \mathrm{GO} / \mathrm{Ag} / \mathrm{AgBr}$ displays distinctly boosted catalytic activity towards the photodegradation of methyl orange (MO) molecules under visible-light irradiation. We disclose that the GO-facilitated good hybridization between $\mathrm{Gr}$ and $\mathrm{Ag} / \mathrm{AgBr}$, and the efficient separation of photogenerated electrons and holes promoted by GO and Gr, play an important role for the enhanced catalytic activity. Taking into account the excellent hybridization capability of GO, its versatile multifunctionality and scalable accessibility, and together with the low-cost of Gr and its exceptional electronic characteristics, we anticipate that our efficient yet simple protocol might be applicable not only to $\mathrm{Ag} / \mathrm{AgBr}$-based plasmonic photocatalysts but also to a wide variety of others materials, wherein Gr/GO-based advanced materials of desired and improved functions might be facilely launched.

\section{Experimental section}

\subsection{Chemicals and materials}

Silver nitrate $\left(\mathrm{AgNO}_{3}, \quad\right.$ Sigma-Aldrich, $\left.>99 \%\right)$, cetyltrimethylammonium bromide (CTAB, Alfa Aesar, 95\%), graphite powder (Gr, Alfa Aesar, 325 mesh, >99.9\%), methyl orange (MO, Alfa Aesar, $>98 \%$ ), ethanol (99.7\%, Beijing Chemical Works), and $\mathrm{NaCl}$ (AR, Beijing Chemical Works) were used as received. P25$\mathrm{TiO}_{2}$ (ca. $80 \%$ anatase and $20 \%$ rutile) species were purchased from Degussa and used as the reference photocatalysts for comparison. Indium tin oxide glasses (ITO) were purchased from Zhuhai Kaivo Optoelectronic Technology Co., Ltd. GO was synthesized via a modified Hummers' method. ${ }^{31}$ Ultrapure Milli$\mathrm{Q}$ water (18.2 $\mathrm{M} \Omega$ ) was used for the preparation of various aqueous dispersions.

\subsection{Pretreatment of $\mathrm{Gr}$ powders and preparation of $\mathrm{Gr} / \mathrm{GO}$}

The original Gr powders ( $1 \mathrm{~g}$ ) were added into a mixture of Milli-Q water $(40 \mathrm{~mL})$ and ethanol $(5 \mathrm{~mL})$. The system was treated with an ultrasonic homogenizer (Ningbo Scientz Biotechnology Co., Ltd., Scientz-II D, frequency $=20 \mathrm{kHz}$, output power $=400 \mathrm{~W}$ ) for 7 hours. During the ultrasonication, the system was maintained below $20{ }^{\circ} \mathrm{C}$ using an ice bath. Then, the suspension was repeatedly treated by centrifugation (11000 rpm, $10 \mathrm{~min}$ ) for 4 times using ethanol as solvent. The obtained precipitates were dried under vacuum at room temperature for 24 hours. For the preparation of the aqueous dispersion of $\mathrm{Gr} / \mathrm{GO}, 20 \mathrm{mg}$ of the aspretreated $\mathrm{Gr}$ powders were added into an aqueous dispersion of GO $\left(20 \mathrm{~mL}, 1 \mathrm{mg} \mathrm{mL} \mathrm{m}^{-1}\right)$. Then, the system was treated with the ultrasonic homogenizer for 3 hours, after which the as-obtained dispersion was used for the preparation of $\mathrm{Gr} / \mathrm{GO} / \mathrm{Ag} / \mathrm{AgBr}$ hybrids.

\subsection{Fabrication of $\mathrm{Gr} / \mathrm{GO} / \mathrm{Ag} / \mathrm{AgBr}, \mathrm{Gr} / \mathrm{Ag} / \mathrm{AgBr}, \mathrm{GO} / \mathrm{Ag} / \mathrm{AgBr}$ and $\mathrm{Ag} / \mathrm{AgBr}$ hybrids}

To prepare $\mathrm{Gr} / \mathrm{GO} / \mathrm{Ag} / \mathrm{AgBr}$ species, $0.5 \mathrm{~mL}$ of the above obtained $\mathrm{Gr} / \mathrm{GO}$ dispersion was added dropwise into an aqueous solution of $\mathrm{AgNO}_{3}\left(10 \mathrm{~mL}, 5 \times 10^{-3} \mathrm{~mol} \mathrm{~L}^{-1}\right)$ under magnetic stirring. The mixture was stirred for 30 minutes, after which a chloroform solution of CATB $\left(0.5 \mathrm{~mL}, 6 \times 10^{4} \mathrm{mg} \mathrm{L}^{-1}\right)$ was injected into the suspension within $c a .5$ minutes under ambient conditions. The system was stirred for 30 minutes for chloroform evaporation. Subsequently, the products was collected via centrifugation (11 $000 \mathrm{rpm}, 10$ minutes), and the obtained solids was washed by a mixture of Milli-Q water and ethanol (1:1) for 1 time, and by Milli-Q water for 3 times. Thus-fabricated solids were then subjected to various characterizations or uses. To prepare $\mathrm{Gr} / \mathrm{Ag} /$ $\mathrm{AgBr}, \mathrm{GO} / \mathrm{Ag} / \mathrm{AgBr}$ and $\mathrm{Ag} / \mathrm{AgBr}$ species, nearly identical operations were conducted except that an aqueous dispersion of $\mathrm{Gr}$ (0.5 mL, $\left.1 \mathrm{mg} \mathrm{mL}^{-1}\right)$, GO $\left(0.5 \mathrm{~mL}, 1 \mathrm{mg} \mathrm{mL}^{-1}\right)$, or Milli-Q water $(0.5 \mathrm{~mL})$, respectively, but not the dispersion of $\mathrm{Gr} / \mathrm{GO}$, was added into the aqueous solution of $\mathrm{AgNO}_{3}$.

\subsection{Photocatalytic performances}

To estimate the photocatalytic activity of the above-prepared species, $9 \mathrm{mg}$ of the respective structures was introduced into 
$9 \mathrm{~mL}$ aqueous solution of $\mathrm{MO}\left(60 \mathrm{mg} \mathrm{L}^{-1}\right)$, where a quartz cuvette was used as photoreactor. For catalytic experiments, a $500 \mathrm{~W}$ xenon arc lamp (CHF-XM35-500 W, Beijing Trusttech Co. Ltd.), which was installed in a laboratory lamp housing system, was used as light source. Before entering the photoreactor, the light passed through a water filter of a thickness of $10 \mathrm{~cm}$ and a UV cutoff filter (>400 nm). The photoreaction system was kept in a dark room for 60 minutes to realize an equilibrium adsorption state before visible-light irradiation. The dark adsorption time was designed to be 60 minutes, since it was found that even when a longer adsorption time of 24 hours was applied, similar results were obtained. Aliquots of the dispersion $(0.3 \mathrm{~mL})$ were taken out from the photoreaction system for real-time sampling. The degradation of MOs was monitored by measuring their real-time UV-vis absorptions at $463 \mathrm{~nm}$. For the evaluation of catalytic reactivity, $C$ was the concentration of $\mathrm{MO}$ at a desired reaction time $t, C_{0}$ was the concentration of the MO solution immediately before it was kept in dark.

\subsection{Photoelectrochemical measurements}

For photoelectrochemical measurements, ITO glass electrodes were modified by our samples. To achieve this, the abovesynthesized materials $(5 \mathrm{mg})$ were dispersed in ethanol (2 $\mathrm{mL})$, and the dispersion was drop-cast onto a pre-cleaned ITO electrode $(2 \mathrm{~cm} \times 4 \mathrm{~cm})$. The as-modified ITO plates were dried under ambient conditions for 48 hours. The photocurrent experiments were conducted in a three-electrode system, where the as-treated ITO electrode, a platinum wire, and a saturated calomel electrode (SCE) were used as working electrode, counter electrode and reference electrode, respectively, while an aqueous solution of $\mathrm{NaCl}(0.1 \mathrm{M})$ was used as electrolyte. During the measurements, the ITO electrode was intermittently irradiated with visible-light, and the photocurrent-time characteristics were recorded with a CHI 660B electrochemical analyzer (Shanghai Chenhua Instrumental Co., Ltd.). The above set-up was also used for the measurement of electrochemical impedance spectra (EIS) of our samples, which were recorded with the help of ZPlot/ZView software under an ac perturbation signal of $5 \mathrm{mV}$ over a frequency range of $0.05 \mathrm{~Hz}$ to $100 \mathrm{kHz}$ at a potential of $0.1 \mathrm{~V}$.

\subsection{Apparatus}

The scanning electron microscopy (SEM) measurements were conducted using a Hitachi S-4800 system. The energy dispersive X-ray spectroscopy (EDX) of the samples was measured with a Horiba EMAX X-act energy dispersive spectroscopy that was attached to the Hitachi S-4800 system. The powder X-ray diffraction (PXRD) measurements were conducted on a PANalytical X'Pert PRO instrument with $\mathrm{Cu} \mathrm{K} \alpha$ radiation. The UV-vis diffuse reflectance spectra of our samples were measured using an UV-vis spectrophotometer (Hitachi U-3010), wherein $\mathrm{BaSO}_{4}$ was used as reference. The degradation of MOs was monitored by measuring their real-time UV-vis spectra using a Hitachi U-3010 spectrometer. The Raman spectra were recorded on a Renishaw inVia plus Raman microscope using a $514.5 \mathrm{~nm}$ argon ion laser. X-ray photoelectron spectroscopy (XPS) was performed on an ESCALab220i-XL electron spectrometer from VG Scientific using $300 \mathrm{~W} \mathrm{Al} \mathrm{K} \alpha$ radiation. The binding energies were referenced to the $\mathrm{C} 1 \mathrm{~s}$ line at $284.8 \mathrm{eV}$ from adventitious carbon.

\section{Results and discussion}

Experimentally, the Gr powders were pre-treated with an ultrasonic homogenizer before uses. As shown in Fig. 1a and b, the as treated Gr powders manifest themselves as irregular sheet-like bulk structures with a lateral dimension of $0.5-50 \mu \mathrm{m}$ and a thickness of $3 \mu \mathrm{m}$ to $50 \mathrm{~nm}$. In practice, we tried to fabricate $\mathrm{Gr} /$ $\mathrm{Ag} / \mathrm{AgBr}$ hybrids via a microemulsion protocol, in which CTAB and $\mathrm{AgNO}_{3}$ were employed as $\mathrm{Br}$ and $\mathrm{Ag}$ sources, respectively. ${ }^{31}$ To fulfill this, an aqueous dispersion of the as-treated Gr was injected dropwise into an aqueous solution of $\mathrm{AgNO}_{3}$ under vigorous stirring and ambient conditions. Then, a chloroform solution of CTAB was introduced into the dispersion. After the evaporation of chloroform, the products were collected and washed adequately with ultrapure Milli-Q water in terms of a couple of repeating centrifugation and redispersion. As reported in Fig. 1c and d, sheet-like bulk Gr structures and $\mathrm{Ag} / \mathrm{AgBr}$ species are observed from the typical SEM images of as-fabricated samples. It should be noted that most of the Ag/AgBr species are evidently separated from the Gr sheets, while a large fraction of the surface of the Gr sheets display similar characteristics as that of the bare Gr powders shown in Fig. 1a and b, despite of the fact that merely a small percentage of the surface is occasionally decorated with $\mathrm{Ag} / \mathrm{AgBr}$ nanospecies. These results suggest that the Gr could not form good composites with $\mathrm{Ag} / \mathrm{AgBr}$ species. This could be a consequence of the lack of hybridizable sites on the surface of Gr, leading to a poor hybridization.
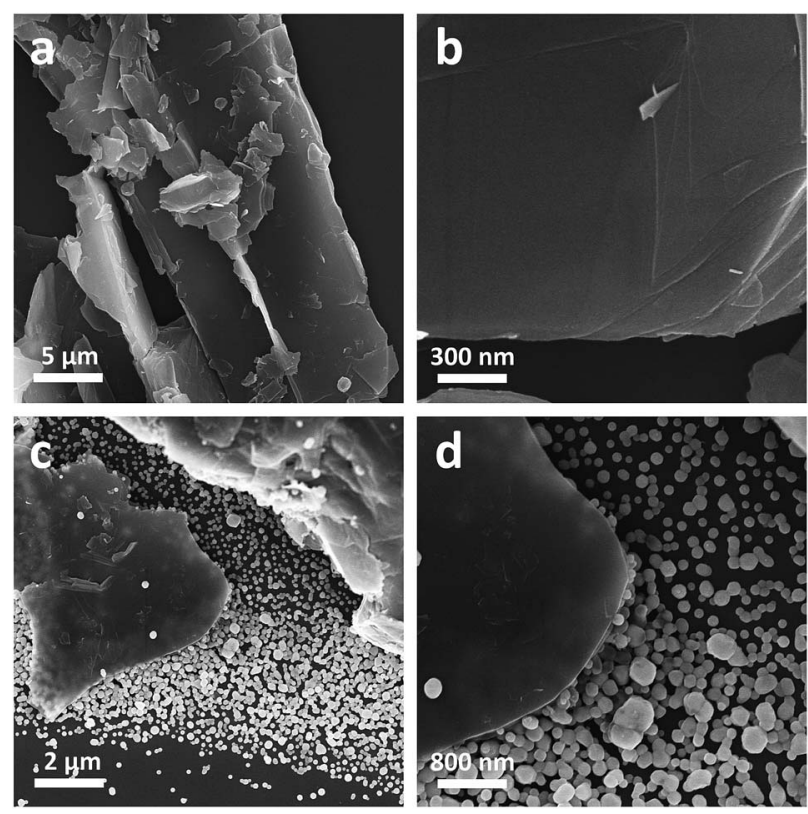

Fig. 1 The typical low-magnification ( $a$ and $c$ ) and high-magnification ( $b$ and d) SEM images of the Gr powders ( $a$ and $b$ ) and the $\mathrm{Gr} / \mathrm{Ag} / \mathrm{AgBr}$ structures. 
Then, we attempted to fabricate $\mathrm{Gr} / \mathrm{GO} / \mathrm{Ag} / \mathrm{AgBr}$ hybrids with an expectation that $\mathrm{Gr}$ could be well hybridized with $\mathrm{Ag} / \mathrm{AgBr}$ by taking the advantage of the excellent hybridization capability of GO nanosheets with $\mathrm{Ag} / \mathrm{AgBr}$ and with $\mathrm{Gr}$ via the oxygencontaining functional groups ${ }^{31,32}$ and the $\pi$-conjugated polyaromatic domains, ${ }^{\mathbf{2 9}, 30}$ respectively. To achieve this, Gr/GO was first prepared by an ultrasonication treatment of a mixture of $\mathrm{Gr}$ and GO. As shown in Fig. 2a and b, the SEM image of thusformulated products shows that sheet-like bulk Gr structures, whose surface are evidently covered with gauze-like GO nanosheets, could be observed, while nearly no free GO nanosheets, which stand away from the Gr structures could be perceived. On the one hand, it should be noted that the as-fabricated structures were collected and washed adequately via centrifugation and resuspension treatments several times. On the other hand, our results show that when the aqueous of GO nanosheets are treated under the similar conditions no precipitates could be observed from the bottom or the wall of the centrifuge tubes, suggesting that GO nanosheets could not be obtained simple by a centrifugation under our experimental conditions. These results basically imply the formation of Gr/GO composites.

This could be verified by the Raman spectra of the samples as shown in Fig. 3, wherein those of the bare GO nanosheets and $\mathrm{Gr}$ powders are also reported for comparison. In the case of GO nanosheets, the characteristic G- and D-bands around 1604 and $1352 \mathrm{~cm}^{-1}$, respectively, could be observed. In addition, a combination of G- and D-bands at ca. $2947 \mathrm{~cm}^{-1}$, and a weakly smeared 2D-band around $2729 \mathrm{~cm}^{-1}$ could also be detected. These features are in accordance with the well-defined Raman spectra of GO nanosheets. ${ }^{48}$ The Raman spectrum of the bare $\mathrm{Gr}$ powders exhibits characteristic strong G-band around 1581 $\mathrm{cm}^{-1}$, weak D-band around $1352 \mathrm{~cm}^{-1}$, and 2D-band at $c a$.
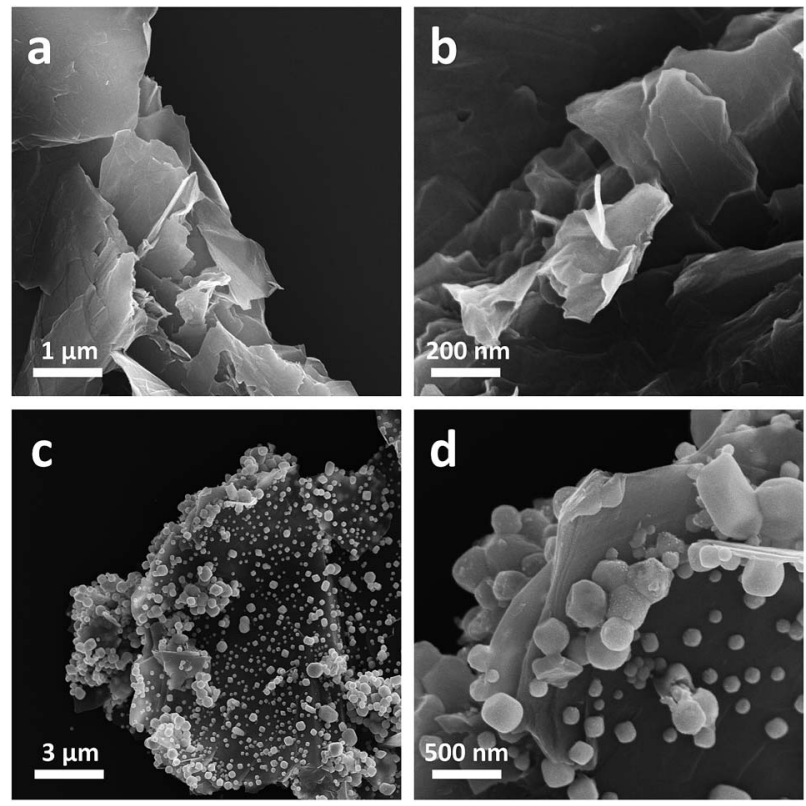

Fig. 2 The typical low-magnification ( $a$ and $\mathrm{c}$ ) and high-magnification ( $b$ and $d$ ) SEM images of the $\mathrm{Gr} / \mathrm{GO}$ ( $a$ and b) and $\mathrm{Gr} / \mathrm{GO} / \mathrm{Ag} / \mathrm{AgBr}$ hybrids.

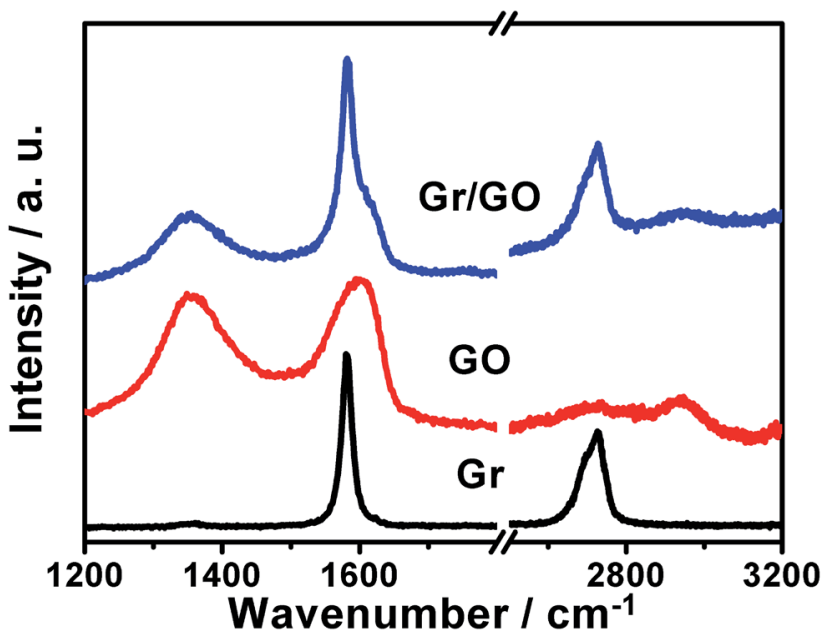

Fig. 3 Raman spectra of Gr (black), GO (red) and Gr/GO (blue) species.

$2722 \mathrm{~cm}^{-1}$, respectively. ${ }^{49}$ In the case of the as-fabricated Gr/GO samples, D-, G-, overtone 2D-, and combination of D + G bands could be observed. Together with the observations of SEM image shown in Fig. 2a and b, these results validate the formation of $\mathrm{Gr} / \mathrm{GO}$ composites, which is induced by the $\pi-\pi$ interactions between these two components. ${ }^{29,30}$

Inspired by the successful fabrication of $\mathrm{Gr} / \mathrm{GO}$ composites, we tried to synthesize $\mathrm{Gr} / \mathrm{GO} / \mathrm{Ag} / \mathrm{AgBr}$ hybrids according to the similar procedures as those of $\mathrm{Gr} / \mathrm{Ag} / \mathrm{AgBr}$. In this case, an aqueous dispersion of $\mathrm{Gr} / \mathrm{GO}$ instead of that of the Gr powders was used. As presented in Fig. $2 c$ and d, it can be seen from the SEM image of the as-fabricated products that $\mathrm{Ag} / \mathrm{AgBr}$ species, which are distributed on the surface of Gr/GO species could be observed. Significantly, it is worth pointing out that only negligible amount of free $\mathrm{Ag} / \mathrm{AgBr}$ species could be observed outside the Gr/GO structures. Accompanied by the results obtained from the $\mathrm{Gr} / \mathrm{Ag} / \mathrm{AgBr}$ systems (Fig. 1c and d), which are synthesized without the involvement of GO nanosheets, and together with the results of Raman spectra shown in Fig. 3, these present observations suggest that Gr could be facilely yet well hybridized with $\mathrm{Ag} / \mathrm{AgBr}$ species under the assistance of GO nanosheets, wherein the anchoring of the $\mathrm{Ag} / \mathrm{AgBr}$ species by the oxygen-containing functional groups of the GO component of the $\mathrm{Gr} / \mathrm{GO}$ composites promotes the formation of the $\mathrm{Gr} / \mathrm{GO}$ / $\mathrm{Ag} / \mathrm{AgBr}$ species. ${ }^{31,32}$ It can be seen that $\mathrm{Ag} / \mathrm{AgBr}$ could not be well hybridized with the pristine Gr without the assistance of GO nanosheets, while a good hybridization could be realized under the assistance of GO. Accordingly, we tentatively suggest that the $\mathrm{Ag} / \mathrm{AgBr}$ species are most likely anchored on the surface of the GO species of the $\mathrm{Gr} / \mathrm{GO} / \mathrm{Ag} / \mathrm{AgBr}$ hybrid, while the possibility of being anchored on the surface of the Gr species might be very low.

In addition to the $\mathrm{Gr} / \mathrm{Ag} / \mathrm{AgBr}$ and $\mathrm{Gr} / \mathrm{GO} / \mathrm{Ag} / \mathrm{AgBr}$ species, $\mathrm{GO} / \mathrm{Ag} / \mathrm{AgBr}$ and $\mathrm{Ag} / \mathrm{AgBr}$ structures (Fig. S1 and $\mathrm{S} 2 \dagger$ ) were also formulated by means of our microemulsion protocol, wherein an aqueous dispersion of GO or pure Milli-Q water, respectively, but not the dispersion of $\mathrm{Gr}$ or $\mathrm{Gr} / \mathrm{GO}$, was used during the synthesis. As described in the following sections, these 
materials were prepared to make a comparative investigation on their catalytic activity with regard to the effects of $\mathrm{GO}, \mathrm{Gr}$, and Gr/GO species.

The components of our $\mathrm{Ag} / \mathrm{AgBr}, \mathrm{Gr} / \mathrm{Ag} / \mathrm{AgBr}, \mathrm{GO} / \mathrm{Ag} / \mathrm{AgBr}$ and $\mathrm{Gr} / \mathrm{GO} / \mathrm{Ag} / \mathrm{AgBr}$ species were investigated by means of energy dispersive X-ray spectroscopy (EDX) analyses. As reported in Fig. $\mathrm{S} 3, \uparrow$ in the case of the bare $\mathrm{Ag} / \mathrm{AgBr}$ structures, only EDX signals attributed to $\mathrm{Ag}$ and $\mathrm{Br}$ elements could be evidently observed. The atomic ratio between $\mathrm{Ag}$ and $\mathrm{Br}$ is semiquantitatively estimated to be $c a$. $1.1: 1$. This value is larger than the theoretic stoichiometric atomic ratio between $\mathrm{Ag}$ and $\mathrm{Br}$ elements of $\mathrm{AgBr}$, which should be $1: 1$. This could be owing to the generation of metallic $\mathrm{Ag}^{\mathrm{O}}$ nanostructures, which is induced by the ambient light during the fabrication procedure, resulting in the formation of $\mathrm{Ag} / \mathrm{AgBr}$ structures. ${ }^{31,32}$ For $\mathrm{Gr} / \mathrm{Ag} /$ $\mathrm{AgBr}$ structures, nearly similar results with an $\mathrm{Ag} / \mathrm{Br}$ ratio of $\mathrm{ca}$. 1.1 : 1 are also obtained except that $\mathrm{C}$ element could also be detected distinctly. The appearance of $\mathrm{C}$ signals is in agreement with the existence of $\mathrm{Gr}$ in the samples. In the cases of the GO/ $\mathrm{Ag} / \mathrm{AgBr}$ and $\mathrm{Gr} / \mathrm{GO} / \mathrm{Ag} / \mathrm{AgBr}$ structures, besides the $\mathrm{Ag}, \mathrm{Br}$ and $\mathrm{C}$ signals, those attributed to $\mathrm{O}$ element could also be observed. This is owing to the presence of GO nanosheets in these systems. Similar as the results of the $\mathrm{Ag} / \mathrm{AgBr}$ and $\mathrm{Gr} / \mathrm{Ag} / \mathrm{AgBr}$ structures, the atomic ratio between $\mathrm{Ag}$ and $\mathrm{Br}$ is also estimated to be $c a .1 .1: 1$. Note that the atomic ratio between $\mathrm{C}$ and $\mathrm{O}$ is ca. 4.9 and 2.8, respectively, for $\mathrm{Gr} / \mathrm{GO} / \mathrm{Ag} / \mathrm{AgBr}$ and $\mathrm{GO} / \mathrm{Ag} /$ $\mathrm{AgBr}$. This result is reasonable, since only $\mathrm{C}$ but negligible $\mathrm{O}$ exists in the skeleton of Gr, which increases and decreases the relative content of $\mathrm{C}$ and $\mathrm{O}$, respectively.

To verify the production of $\mathrm{Ag} / \mathrm{AgBr}$-based species, our samples were investigated by means of powdery XRD (PXRD). As present in Fig. 4, all of our samples display distinct diffraction peaks $(2 \theta)$ at $c a .26 .9^{\circ}, 31.1^{\circ}, 44.4^{\circ}, 52.7^{\circ}, 55.2^{\circ}, 64.7^{\circ}$ and $73.4^{\circ}$, which could be well indexed to the typical diffractions of the (111), (200), (220), (311), (222), (400) and (331) facets of cubic phase AgBr (JCPDS file: 6-438). ${ }^{33,34}$ At the same time, a weak yet

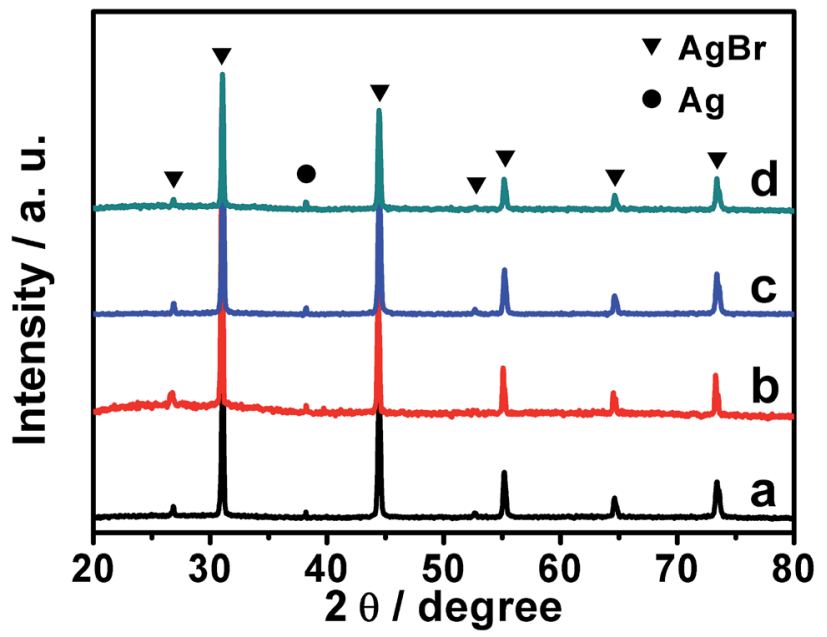

Fig. 4 The PXRD patterns of our $\mathrm{Ag} / \mathrm{AgBr}(\mathrm{a}), \mathrm{GO} / \mathrm{Ag} / \mathrm{AgBr}(\mathrm{b}), \mathrm{Gr} / \mathrm{Ag} /$ $\mathrm{AgBr}$ (c) and $\mathrm{Gr} / \mathrm{GO} / \mathrm{Ag} / \mathrm{AgBr}$ (d) structures. The diffraction peaks indexed to $\mathrm{AgBr}$ and $\mathrm{Ag}^{\mathrm{O}}$ species are marked with $\boldsymbol{\nabla}$ and respectively. distinct diffraction peak around $38.3^{\circ}$, which could be ascribed to the (111) facet of cubic phase metallic Ag (JCPDS file: 65$2871),{ }^{33}$ could also be observed. Together with the results of EDX analyses shown in Fig. S3, $\uparrow$ these experimental facts validate the formation of $\mathrm{Ag} / \mathrm{AgBr}$ species in our samples. It should be pointed out that negligible diffraction peaks ascribing to the GO or Gr could be observed from the PXRD patterns of our GO/ $\mathrm{Ag} / \mathrm{AgBr}, \mathrm{Gr} / \mathrm{Ag} / \mathrm{AgBr}$ and $\mathrm{Gr} / \mathrm{GO} / \mathrm{Ag} / \mathrm{AgBr}$ samples. This is a result of their low content. ${ }^{50,51}$

For visible-light-driven photocatalysts, it is essentially necessary that they should be visible-light active so as to fulfill an efficient energy supply and a high catalytic performance. The UV-visible diffuse reflectance spectra of our samples were examined, as shown in Fig. 5. It can be seen that besides the evident absorptions in the ultraviolet region, which could be ascribed to the AgBr species, broad and strong absorptions in the visible region in the range of $460-800 \mathrm{~nm}$ could also be distinctly observed. Generally, AgBr species could only exhibit evident absorptions in ultraviolet region but slight absorptions in visible region. ${ }^{33-35}$ Accompanied by the results of EDX, and PXRD presented in Fig. S3 and S4, $\dagger$ respectively, these results further indicate the existence of metallic $\mathrm{Ag}^{\mathrm{O}}$ species in our products, which can arouse surface plasmon resonance (SPR) absorptions in visible region. ${ }^{36-38,52}$ As known, the plasmonic absorption of metallic $\mathrm{Ag}$ nanoarchitectures depends, to a great extent, on their size, shape etc., and those containing Ag nanostructures of diverse shapes or sizes could generally exhibit a broad SPR peak. ${ }^{37,38,52}$ Similar broad SPR absorptions in the visible region have been reported previously for the UV-vis spectra of $\mathrm{Ag} / \mathrm{AgBr}$-based materials. ${ }^{33,36}$ Experimentally, we attempted to disclose the shape and size of the metallic $\mathrm{Ag}^{0}$ structures of our samples via transmission electron microscopy (TEM). It is unfortunately found that the samples suffered a fast decomposition immediately after the focusing of the electron beam. This makes it difficult to identify the size and shape of $\mathrm{Ag}^{0}$ of the $\mathrm{Ag} / \mathrm{AgBr}$ structures via TEM. Similar results has been reported previously for silver halide-based plasmonic photocatalysts. ${ }^{39-41}$ Nevertheless, it could be suggested that the broad

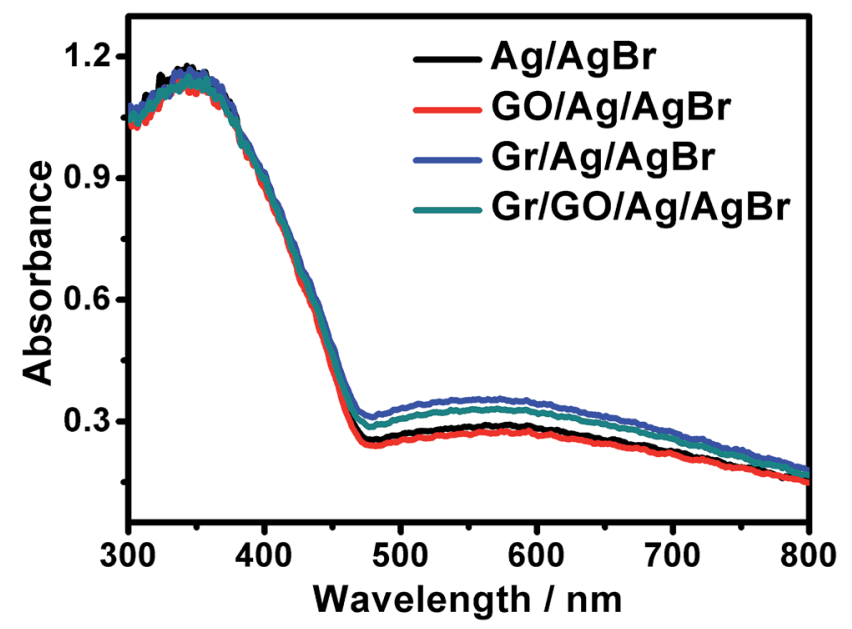

Fig. 5 The UV-visible diffuse reflectance spectra of our $\mathrm{Ag} / \mathrm{AgBr}$ based materials. 
SPR peaks observed from our samples could be due to the presence of $\mathrm{Ag}^{0}$ nanospecies of various sizes and shapes. ${ }^{36-38,52}$

Motivated by the above-mentioned results, the photocatalytic performances of our $\mathrm{Ag} / \mathrm{AgBr}$-based structures toward the degradation of MO molecules under visible-light irradiations were studied. As plotted in Fig. 6a (magenta curve), when the photoreaction was conducted without the presence of our $\mathrm{Ag} / \mathrm{AgBr}$-based materials, negligible photodegradation of MOs could be observed, implying that the self-photosensitized bleaching of MOs under our experimental conditions could basically be ignored. When the bare $\mathrm{Ag} / \mathrm{Ag} \mathrm{Br}$ structures are introduced into the photoreaction system, $c a$. 46.9\% MOs are eliminated within 40 minutes under the similar experimental conditions (Fig. 6a, black curve), indicating that that our $\mathrm{Ag} /$ AgBr could work as visible-light-driven photocatalysts for the photodegradation of MOs. ${ }^{33,34,36-38}$ On the other hand, when the as-fabricated $\mathrm{GO} / \mathrm{Ag} / \mathrm{AgBr}$ and $\mathrm{Gr} / \mathrm{Ag} / \mathrm{AgBr}$ structures are
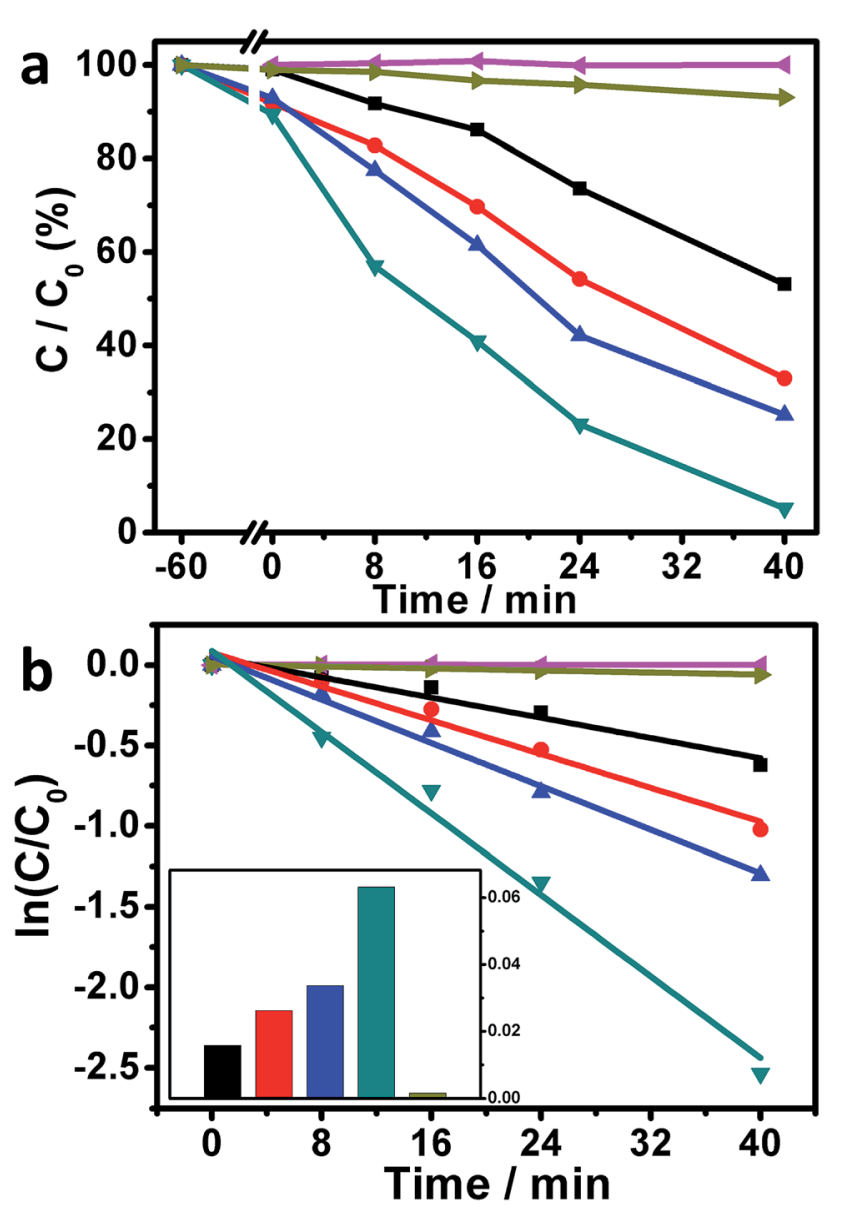

Fig. 6 Photocatalytic performances (a), the corresponding kinetic linear simulation curves (b), and the histogram of the photoreaction rate constants (inset of b) of $\mathrm{Ag} / \mathrm{AgBr}$ (black), GO/Ag/AgBr (red), $\mathrm{Gr} / \mathrm{Ag} /$ $\mathrm{AgBr}$ (blue) and $\mathrm{Gr} / \mathrm{GO} / \mathrm{Ag} / \mathrm{AgBr}$ (dark cyan) structures. The results of a blank experiment (magenta curves) and a controlled (dark yellow curves) experiment, wherein no catalysts and $\mathrm{P} 25-\mathrm{TiO}_{2}$ species are used, respectively, are also presented in the corresponding panels for comparison. The $C / C_{0}$ located at -60 and 0 minutes are the results obtained before and after the dark adsorption experiments, respectively, which are conducted in a dark room without light irradiation for 60 minutes. employed as photocatalysts, ca. $67.0 \%$ (Fig. 6a, red curve) and 74.8\% (Fig. 6a, blue curve) MOs are decomposed, respectively, while this value is further increased to as high as $94.8 \%$ (Fig. 6a, dark cyan curve) when our $\mathrm{Gr} / \mathrm{GO} / \mathrm{Ag} / \mathrm{AgBr}$ species are introduced into the photoreaction system, as shown in Fig. 6a and summarized in Table 1 . The rate constants of the photocatalytic performances of our Ag/AgBr-based materials could be derived by a kinetic linear simulation of the photocatalytic curves in terms of the correlation between $\ln \left(C / C_{0}\right)$ and the reaction time $(t)$. It can be seen from Fig. $6 \mathrm{~b}$ that there is a good linear correlation between $\ln \left(C / C_{0}\right)$ and $t$. This indicates that the photodegradation reaction of MOs over our $\mathrm{Ag} / \mathrm{AgBr}$-based photocatalysts follows the first-order kinetics: $-\mathrm{d} C / \mathrm{d} t=k C$, wherein $C$ represents the concentration of MOs at a real-time, $t$ and $k$ stand for reaction time and rate constant, respectively. ${ }^{41}$

As shown in Fig. 6b and summarized in Table 1, the rate constants of the photocatalytic degradation of MOs over our Ag/ $\mathrm{AgBr}, \mathrm{GO} / \mathrm{Ag} / \mathrm{AgBr}, \mathrm{Gr} / \mathrm{Ag} / \mathrm{AgBr}$, and $\mathrm{Gr} / \mathrm{GO} / \mathrm{Ag} / \mathrm{AgBr}$ species are estimated to be $0.016,0.026,0.034$, and $0.063 \mathrm{~min}^{-1}$, respectively, wherein an increasing tendency is obtained. It can be seen that compared to the $\mathrm{Ag} / \mathrm{AgBr}, \mathrm{GO} / \mathrm{Ag} / \mathrm{AgBr}$, and $\mathrm{Gr} / \mathrm{Ag} /$ $\mathrm{AgBr}$ counterparts, the catalytic activity of our $\mathrm{Gr} / \mathrm{GO} / \mathrm{Ag} / \mathrm{AgBr}$ hybrids is evidently enhanced by a factor of 3.9, 2.4, and 1.9 times, respectively. These results suggest that among our Ag/ AgBr-based structures, the $\mathrm{Gr} / \mathrm{GO} / \mathrm{Ag} / \mathrm{AgBr}$ hybrids are relatively superior visible-light-driven photocatalysts towards the photobleaching of MOs. Moreover, the photoreaction was also conducted by using the commercially available $\mathrm{P} 25-\mathrm{TiO}_{2}$ as the photocatalyst. The results show that only ca. 6.9\% MOs are bleached under the similar experimental conditions with a rate constant of as low as ca. $0.0016 \mathrm{~min}^{-1}$ (Fig. 6a and b, dark yellow curves). These values are evidently smaller than the corresponding data obtained from the photoreactions systems, wherein our Ag/AgBr-based species were employed as photocatalysts. This indicates the distinct advantages of our $\mathrm{Ag} / \mathrm{AgBr}-$ based structures over the $\mathrm{P} 25-\mathrm{TiO}_{2}$.

On the one hand, it is widely known that the separation/ transfer capability of photogenerated electrons and holes is one of the most important and fundamental issues that could affect the catalytic reactivity of semiconductor-based photocatalysts, wherein the more efficient of the electron-hole separation/ transfer, the higher the catalytic activity of the catalysts, and vice versa..$^{36-38,53}$ On the other hand, it has been demonstrated that the catalytic reactivity of semiconductor photocatalysts could be improved to some extent when they are hybridized with GO nanosheets. ${ }^{\text {9,11-15,17 }}$ This could be owing to the GO-promoted separation/transfer of the photogenerated electrons and holes., ${ }^{\mathbf{9 1 1 - 1 5 , 1 7}}$ In spite of the fact that GO nanosheets exhibit substantially depressed electron transport characteristic compared to the pristine graphene, their polyaromatic graphene segment could serve as an electron relay mediator to facilitate the charge separation/transfer, resulting in a boosted photocatalytic performance..$^{\mathbf{9 1 1 - 1 5 , 1 7}}$ To validate this issue in our present case, the transient photocurrent response behaviors of our $\mathrm{Ag} / \mathrm{AgBr}$ and $\mathrm{GO} / \mathrm{Ag} / \mathrm{AgBr}$ species were examined with regard to their photocurrent-time response $(I-t)$ curves, which were measured by means of a three-electrode photoelectrochemical cell. During the 
Table 1 Summary of the catalytic performances of our $\mathrm{Ag} / \mathrm{AgBr}$ based hybrids towards the photodegradation of MOs under visiblelight illumination

\begin{tabular}{llll}
\hline Catalyst & $\begin{array}{l}\text { Degradation } \\
\text { percentage } \\
(\%)\end{array}$ & $\begin{array}{l}\text { Kinetic } \\
\text { constant } \\
\left(\mathrm{min}^{-1}\right)\end{array}$ & $\begin{array}{l}\text { Determination } \\
\text { coefficient } \\
\left(R^{2}\right)\end{array}$ \\
\hline $\mathrm{Ag} / \mathrm{AgBr}$ & 46.9 & 0.016 & 0.949 \\
$\mathrm{Gr} / \mathrm{Ag} / \mathrm{AgBr}$ & 67.0 & 0.026 & 0.971 \\
$\mathrm{GO} / \mathrm{Ag} / \mathrm{AgBr}$ & 74.8 & 0.034 & 0.987 \\
$\mathrm{Gr} / \mathrm{GO} / \mathrm{Ag} / \mathrm{AgBr}$ & 94.8 & 0.063 & 0.985 \\
\hline
\end{tabular}

measurements, the indium tin oxide (ITO) glasses, which were covered by our $\mathrm{Ag} / \mathrm{AgBr}$-based materials, were used as the working electrodes and were intermittently irradiated by visiblelight. As illustrated in Fig. 7 (black and red curves), our results show that the photocurrents of the ITO electrodes covered both by our $\mathrm{Ag} / \mathrm{AgBr}$ and $\mathrm{GO} / \mathrm{Ag} / \mathrm{AgBr}$ species display an evident increase immediately when the light irradiation is switched on. In contrast, the intensity of the photocurrent recovers to its original value when the light irradiation is switched off. Such reversible photocurrent response behaviors could be reproducibly and repeatedly conducted over a couple of light switched-on/ off cycles, implying the separation/transportation of the photogenerated electrons and holes in our $\mathrm{Ag} / \mathrm{AgBr}$ and $\mathrm{GO} / \mathrm{Ag} / \mathrm{AgBr}$ structures upon light irradiations. At the same time, we note that for the ITO electrodes covered by our bare $\mathrm{Ag} / \mathrm{AgBr}$ species (Fig. 7, black curve), relatively unconspicuous photocurrent responses with weak photocurrent intensity could be observed. In contrast, in the case of the ITO electrodes modified by our GO/ $\mathrm{Ag} / \mathrm{AgBr}$ structures (Fig. 7, red curve), much more perceptible photocurrent responses of a distinctly enhanced photocurrent intensity could be detected. These observations are in good accordance to our photocatalytic results (Fig. 6 and Table 1) that the compared to the bare $\mathrm{Ag} / \mathrm{AgBr}$ species, the $\mathrm{GO} / \mathrm{Ag} / \mathrm{AgBr}$ counterparts are superior photocatalysts, wherein the GOpromoted separation of photogenerated electrons and holes play an important role..$^{54,55}$

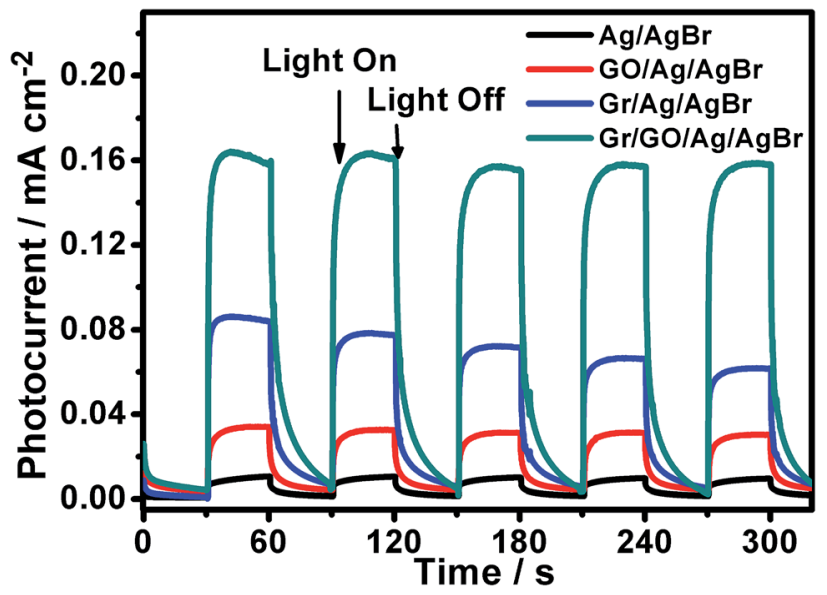

Fig. 7 The transient photocurrent responses of our $\mathrm{Ag} / \mathrm{AgBr}$-based structures. The light irradiation was periodically interrupted every 30 seconds during the measurements.
As addressed in the introduction section, $\mathrm{Gr}$ is structurally composed of coherently superimposed layers of pristine graphene sheets, in which the intact $\pi$-delocalized structure resulted from the $\mathrm{sp}^{2}$-hybridized carbon atoms, provides it with a good electron mobility of as large as $1.2 \times 10^{4} \mathrm{~cm}^{2} \mathrm{~V}^{-1} \mathrm{~s}^{-1} .{ }^{28}$ Such unique characteristics might make Gr excellent electron collector in photocatalysts to accept and transport the photogenerated electrons so as to promote a boosted photocatalytic performance, although the lack of enough hybridizable sites on its surface disfavors its hybridization with $\mathrm{Ag} / \mathrm{AgBr}$ species (Fig. 1). As shown in Fig. 7 (blue curve), compared to those of the bare $\mathrm{Ag} / \mathrm{AgBr}$ and $\mathrm{GO} / \mathrm{Ag} / \mathrm{AgBr}$ species, the ITO electrodes covered by our $\mathrm{Gr} / \mathrm{Ag} / \mathrm{AgBr}$ structures exhibit more obvious transient photocurrent responses with a further increased photocurrent intensity. These facts are in agreement with our catalytic results shown in Fig. 6 and summarized in Table 1, wherein compared to their $\mathrm{Ag} / \mathrm{AgBr}$ and $\mathrm{GO} / \mathrm{Ag} / \mathrm{AgBr}$ counterparts, $\mathrm{Gr} / \mathrm{Ag} / \mathrm{AgBr}$ displays a further improved catalytic activity. This suggests that despite of its poor hybridization capability with $\mathrm{Ag} / \mathrm{AgBr}$ (Fig. 1), Gr could promote the spatial separation/ transfer of the photogenerated electrons and holes of the $\mathrm{Ag} /$ $\mathrm{AgBr}$ species, leading to the enhanced photocatalytic performances of the $\mathrm{Gr} / \mathrm{Ag} / \mathrm{AgBr}$ structures.

Based on the above mentioned experimental facts and analyses, it accordingly could be proposed that the separation/ transfer efficiency of the photogenerated electrons and holes might be further promoted in the case of our $\mathrm{Gr} / \mathrm{GO} / \mathrm{Ag} / \mathrm{AgBr}$ hybrids, since the Gr powders in this case are well hybridized with $\mathrm{Ag} / \mathrm{AgBr}$ under the assistance of GO nanosheets (Fig. 2), and both of GO nanosheets and Gr could play a role of electron relay mediator to promote the charge separation/transfer (Fig. 7, red and blue curves). As shown in Fig. 7 (dark cyan curve), compared to their counterparts of the $\mathrm{Ag} / \mathrm{AgBr}, \mathrm{GO} / \mathrm{Ag} / \mathrm{AgBr}$, and $\mathrm{Gr} / \mathrm{Ag} /$ $\mathrm{AgBr}$, the ITO electrodes covered by the $\mathrm{Gr} / \mathrm{GO} / \mathrm{Ag} / \mathrm{AgBr}$ species display even more prominent transient photocurrent responses, from which an substantially enhanced photocurrent intensity could be observed. These results are essentially consistent with the fact that among our $\mathrm{Ag} / \mathrm{AgBr}$-based visible-light-energized photocatalysts, the $\mathrm{Gr} / \mathrm{GO} / \mathrm{Ag} / \mathrm{AgBr}$ species display the highest catalytic reactivity (Fig. 6 and Table 1). Accompanied by the results obtained from the $\mathrm{GO} / \mathrm{Ag} / \mathrm{AgBr}$ and $\mathrm{Gr} / \mathrm{Ag} / \mathrm{AgBr}$ systems, this confirms that the synergistic effect of $\mathrm{GO}$ and $\mathrm{Gr}$, wherein the former favors a good hybridization between $\mathrm{Ag} / \mathrm{AgBr}$ and $\mathrm{Gr},{ }^{31,32}$ while both the former and the latter promote the separation/ transfer of photogenerated electrons and holes, ${ }^{9,11-15,17}$ contribute much to the observed significantly boosted photocatalytic performances of the $\mathrm{Gr} / \mathrm{GO} / \mathrm{Ag} / \mathrm{AgBr}$ species.

These proposals could be further experimentally corroborated by means of an investigation on the electrochemical impedance spectra (EIS) of our $\mathrm{Ag} / \mathrm{AgBr}$-based structures, which were measured with respect to Nyquist plots. As illustrated in Fig. 8, it can be seen that the EIS of the ITO electrodes covered by our $\mathrm{Ag} / \mathrm{AgBr}, \mathrm{GO} / \mathrm{Ag} / \mathrm{AgBr}$ and $\mathrm{Gr} / \mathrm{Ag} / \mathrm{AgBr}$ structures display a gradually decrease tendency in the radius of their semicircular arcs. Commonly, a smaller semicircular arc in the EIS implies a faster interfacial charge transfer and a more effective separation of the photogenerated electrons and holes..$^{50,56,57}$ These 
results indicate a decrease in the solid state interface layer resistance and the charge transfer resistance on the surface of $\mathrm{Ag} / \mathrm{AgBr}, \mathrm{GO} / \mathrm{Ag} / \mathrm{AgBr}$, and $\mathrm{Gr} / \mathrm{Ag} / \mathrm{AgBr}$ species gradually. ${ }^{50,55,56}$ Accompanied by the aforementioned results of transient photocurrent responses of the corresponding samples shown in Fig. 7 (black, red and blue curves), this further confirms that GO nanosheets and Gr could in faith play a role of electron reservoirs to facilitate charge separation and thus suppress the recombination of the photogenerated electrons and holes, resulting in enhanced catalytic performances. Significantly, it is found that the arc radius of the Nyquist plots of the electrodes cover by our $\mathrm{Gr} / \mathrm{GO} / \mathrm{Ag} / \mathrm{AgBr}$ hybrids exhibit a further decrease compared to those modified by our $\mathrm{GO} / \mathrm{Ag} / \mathrm{AgBr}$ and $\mathrm{Gr} / \mathrm{Ag} / \mathrm{Ag} \mathrm{Br}$ structures. This verifies a more efficient charge separation in the $\mathrm{Gr} / \mathrm{GO} / \mathrm{Ag} / \mathrm{AgBr}$ species, wherein the GO-medicated good hybridization between $\mathrm{Gr}$ and $\mathrm{Ag} / \mathrm{AgBr}$, and the synergistic effect of GO and Gr play an important role.

The XPS spectra of the bare $\mathrm{Ag} / \mathrm{AgBr}$ and $\mathrm{Gr} / \mathrm{GO} / \mathrm{Ag} / \mathrm{AgBr}$ species were also investigated, as shown in Fig. S4. $\dagger$ In the case of the bare $\mathrm{Ag} / \mathrm{AgBr}$ structures, two bands at $c a .373 .4$ and $367.4 \mathrm{eV}$, which could be ascribed to $\mathrm{Ag} 3 \mathrm{~d}_{3 / 2}$ and $\mathrm{Ag} 3 \mathrm{~d}_{5 / 2}$ binding energies, respectively, could be detected (Fig. S4a $\dagger$ ). These two bands could be further deconvoluted into two groups of peaks at $373.4,374.2 \mathrm{eV}$ and $367.4,368.3 \mathrm{eV}$, respectively. The bands located at 374.2 and $368.3 \mathrm{eV}$ are attributed to the metallic $\mathrm{Ag}^{0}$ of $\mathrm{Ag} / \mathrm{AgBr}$, while those located at 373.4 and $367.4 \mathrm{eV}$ are attributed to $\mathrm{Ag}^{+} \cdot{ }^{31,32,41-47}$ It is interesting to note that compared to those of the bare $\mathrm{Ag} / \mathrm{AgBr}$ species, these bands shift to higher binding energies in the XPS spectra of the $\mathrm{Gr} / \mathrm{GO} /$ $\mathrm{Ag} / \mathrm{AgBr}$ hybrids (Fig. S4a $\dagger$ ). Together with the results of the transient photocurrent responses (Fig. 7) and the EIS spectra (Fig. 8) of the corresponding samples, this evident shift in the XPS spectra further indicate that the $\mathrm{Ag} / \mathrm{AgBr}$ species of the $\mathrm{Gr} /$ $\mathrm{GO} / \mathrm{Ag} / \mathrm{AgBr}$ hybrids work as electron donor during the photocatalytic performance, ${ }^{31,32}$ leading to a facilitated charge separation and an enhanced photocatalytic performance (Fig. 6). On the other hand, as shown in Fig. S4b, $\dagger$ the XPS spectra of the $\mathrm{Br}$ species of these samples display binding energy of $\mathrm{Br}_{3 / 2}$ and $\operatorname{Br} 3 \mathrm{~d}_{5 / 2}$ at about 68.1 and $69.0 \mathrm{eV},{ }^{43,45,47}$ respectively. On the

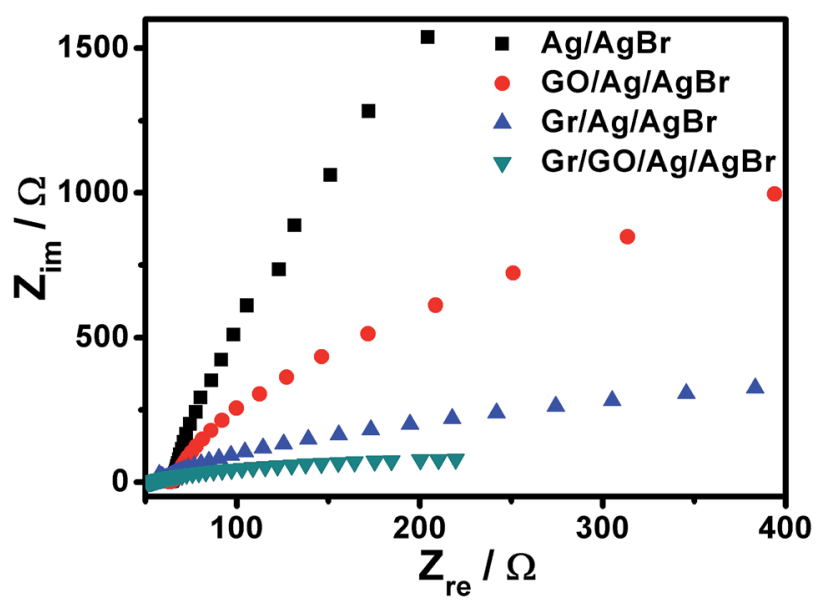

Fig. 8 The typical EIS spectra of the ITO electrodes modified by our $\mathrm{Ag} / \mathrm{AgBr}$-based species.

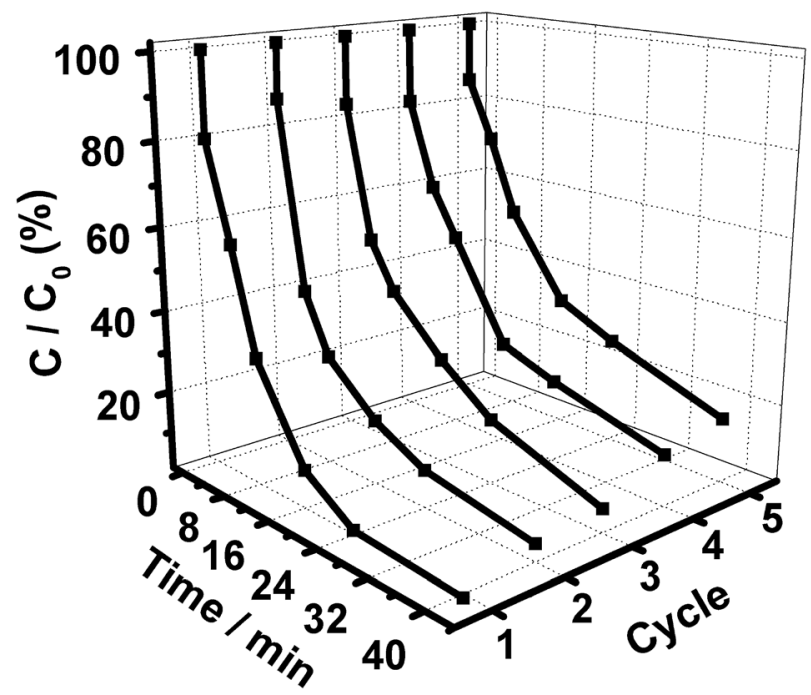

Fig. 9 The consecutive cycling photocatalytic behaviors of $\mathrm{Gr} / \mathrm{GO}$ / $\mathrm{Ag} / \mathrm{AgBr}$ towards the degradation of MOs.

basis of these XPS spectra, the mole ratio between $\mathrm{Ag}$ and $\mathrm{Br}$ elements, and between $\mathrm{Ag}^{0}$ and $\mathrm{Ag}^{+}$could be semiquantitatively evaluated to be $c a$. $1.1: 1$, and $1: 10.2$, respectively, both for the bare $\mathrm{Ag} / \mathrm{AgBr}$ and $\mathrm{Gr} / \mathrm{GO} / \mathrm{Ag} / \mathrm{AgBr}$ structures. These results are in accordance with those of EDX (Fig. S3 $\dagger$ ), wherein the content of $\mathrm{Ag}^{0}$ in the $\mathrm{Gr} / \mathrm{GO} / \mathrm{Ag} / \mathrm{AgBr}$ hybrid could be semiquantitatively assessed to be $c a .4 .6 \%$.

As known, the adsorptive capacity of a catalyst is another factor that could affect its catalytic activity. As shown in Fig. 6a, the adsorptive capacity of our $\mathrm{Ag} / \mathrm{AgBr}$-based structures towards the MO molecules follows $\mathrm{Ag} / \mathrm{AgBr}<\mathrm{GO} / \mathrm{Ag} / \mathrm{AgBr} \approx \mathrm{Gr} / \mathrm{Ag} / \mathrm{AgBr}<$ $\mathrm{Gr} / \mathrm{GO} / \mathrm{Ag} / \mathrm{AgBr}$. This could be attributed to the involvement of $\mathrm{GO}, \mathrm{Gr}$, and $\mathrm{Gr} / \mathrm{GO}$ species in the latter three cases, wherein the noncovalent intermolecular $\pi-\pi$ interactions between the MO molecules and the involved carbonaceous materials promote the adsorption of MOs..31,50 These facts indicate that the good adsorptive capacity of our $\mathrm{Gr} / \mathrm{GO} / \mathrm{Ag} / \mathrm{AgBr}$ structures might also contribute partially to their evidently enhanced photocatalytic activity.

In addition to a high catalytic performance, the durability of a photocatalyst is another important criterion required by excellent catalysts. The recyclability of our $\mathrm{Gr} / \mathrm{GO} / \mathrm{Ag} / \mathrm{AgBr}$ plasmonic photocatalyst was also evaluated in terms of conducting the catalytic performances repeatedly a couple of times. As shown in Fig. 9, the catalytic activity displays only a slight decrease after the catalytic performances are conducted five times continuously. These results suggest that our Gr/GO/Ag/ AgBr hybrids might be used as highly efficient yet stable visible-light-driven plasmonic photocatalysts, indicating its bright future for potential uses.

\section{Conclusions}

In summary, we herein demonstrate that Gr, which originally has a poor hybridization capability owing to the lack hybridizable sites on its skeleton, could be easily and well hybridized 
with $\mathrm{Ag} / \mathrm{AgBr}$-based plasmonic photocatalysts with the assistance of GO nanosheets under ambient conditions. We show that, compared to the counterparts of the bare $\mathrm{Ag} / \mathrm{AgBr}, \mathrm{GO} / \mathrm{Ag} /$ $\mathrm{AgBr}$ and $\mathrm{Gr} / \mathrm{Ag} / \mathrm{AgBr}$ species, the as-synthesized $\mathrm{Gr} / \mathrm{GO} / \mathrm{Ag} / \mathrm{AgBr}$ composites display evidently enhanced photocatalytic activity towards the photodegradation of MOs under visible-light irradiations. Our results indicate that the good hybridization between $\mathrm{Gr}$ and $\mathrm{Ag} / \mathrm{AgBr}$, which is facilitated by GO nanosheets, and the promoted separation of photogenerated electrons and holes, which is favored both by GO and Gr, contribute much to the boosted photocatalytic performances of our $\mathrm{Gr} / \mathrm{GO} / \mathrm{Ag} / \mathrm{AgBr}$ species. On the one hand, on account of the excellent hybridization capacity of GO nanosheets, and the great achievements accumulated in the field of GO-based hybrids, our protocol might not only be applicable to the hybridization of $\mathrm{Gr}$ with $\mathrm{Ag}$ / AgBr-based plasmonic photocatalysts but also to other functional species. On the other hand, considering the good $\pi$ conjugated system of $\mathrm{Gr}$, which make it of great interest in material science, our protocol might provide $\mathrm{Gr}$, which is among the most conventional, low cost, yet important carbonaceous materials, with new and varied opportunities for the construction of emerging advanced hybrid materials.

\section{Acknowledgements}

We acknowledge the financial support from the National Natural Science Foundation of China (Grants 21673253, 21372225, 20873159, 21321063, and 91027042), the National Key Basic Research Project of China (Grants 2013CB834504 and 2011CB932301), and the Chinese Academy of Sciences (Grants XDA09030200, XDB12020200 and 1731300500015).

\section{Notes and references}

1 J. Coraux, L. Marty, N. Bendiab and V. Bouchiat, Acc. Chem. Res., 2012, 46, 2193-2201.

2 A. Mazid Munshi and H. Weman, Phys. Status Solidi RRL, 2013, 7, 713-726.

3 Z. Bo, S. Mao, Z. J. Han, K. Cen, J. Chen and K. Ostrikov, Chem. Soc. Rev., 2015, 44, 2108-2121.

4 J. Park and M. Yan, Acc. Chem. Res., 2013, 46, 181-189.

5 W. I. Park, C.-H. Lee, J. M. Lee, N.-J. Kim and G.-C. Yi, Nanoscale, 2011, 3, 3522-3533.

6 C. K. Chua and M. Pumera, Chem. Soc. Rev., 2013, 42, 32223233.

7 W. Jie and J. Hao, Nanoscale, 2014, 6, 6346-6362.

8 Y. Xu, G. Shi and X. Duan, Acc. Chem. Res., 2015, 48, 16661675.

9 N. Zhang, M.-Q. Yang, S. Liu, Y. Sun and Y.-J. Xu, Chem. Rev., 2015, 115, 10307-10377.

10 S. Mao, G. Lu and J. Chen, Nanoscale, 2015, 7, 6924-6943.

11 N. Gao and X. Fang, Chem. Rev., 2015, 115, 8294-8343.

12 X. Fan, G. Zhang and F. Zhang, Chem. Soc. Rev., 2015, 44, 3023-3035.

13 F. Perreault, A. F. de Faria and M. Elimelech, Chem. Soc. Rev., 2015, 44, 5861-5896.
14 V. Georgakilas, J. N. Tiwari, K. C. Kemp, J. A. Perman, A. B. Bourlinos, K. S. Kim and R. Zboril, Chem. Rev., 2016, 116, 5464-5519.

15 X. Huang, X. Qi, F. Boey and H. Zhang, Chem. Soc. Rev., 2012, 41, 666-686.

16 C. L. Su and K. P. Loh, Acc. Chem. Res., 2013, 46, 2275-2285.

17 Q. Xiang, J. Yu and M. Jaroniec, Chem. Soc. Rev., 2012, 41, 782-796.

18 I. Levchenko, K. Ostrikov, J. Zheng, X. Li, M. Keidar and K. Teo, Nanoscale, 2016, 8, 10511-10527.

19 D. Geng, H. Wang and G. Yu, Adv. Mater., 2015, 27, 28212837.

20 A. V. Zaretski and D. J. Lipomi, Nanoscale, 2015, 7, 99639969.

21 J. N. Coleman, Acc. Chem. Res., 2013, 46, 14-22.

22 T. Kuila, A. K. Mishra, P. Khanra, N. H. Kim and J. H. Lee, Nanoscale, 2013, 5, 52-71.

23 O. C. Compton and S. T. Nguyen, Small, 2010, 6, 711-723.

24 S. Thakur and N. Karak, Carbon, 2015, 94, 224-242.

25 C. K. Chua and M. Pumera, Chem. Soc. Rev., 2014, 43, 291312.

26 R. Sengupta, M. Bhattacharya, S. Bandyopadhyay and A. K. Bhowmick, Prog. Polym. Sci., 2011, 36, 638-670.

27 D. Appy, H. Lei, C.-Z. Wang, M. C. Tringides, D.-J. Liu, J. W. Evans and P. A. Thiel, Prog. Surf. Sci., 2014, 89, 219-238. 28 F. L. Vogel, Synth. Met., 1979/1980, 1, 279-286.

29 J. Kim, L. J. Cote, F. Kim, W. Yuan, K. R. Shull and J. X. Huang, J. Am. Chem. Soc., 2010, 132, 8180-8186.

30 J. Kim, L. J. Cote and J. Huang, Acc. Chem. Res., 2012, 45, 1356-1364.

31 M. Zhu, P. Chen and M. Liu, ACS Nano, 2011, 5, 4529-4536. 32 M. Zhu, P. Chen and M. Liu, Langmuir, 2012, 28, 3385-3390.

33 P. Wang, B. Huang, X. Zhang, X. Qin, H. Jin, Y. Dai, Z. Wang, J. Wei, J. Zhan, S. Wang, J. Wang and M.-H. Whangbo, Chem.-Eur. J., 2009, 15, 1821-1824.

34 B. Li, H. Wang, B. Zhang, P. Hu, C. Chen and L. Guo, ACS Appl. Mater. Interfaces, 2013, 5, 12283-12287.

35 H. Cheng, B. Huang, P. Wang, Z. Wang, Z. Lou, J. Wang, X. Qin, X. Zhang and Y. Dai, Chem. Commun., 2011, 47, 7054-7056.

36 Z. Lou, Z. Wang, B. Huang and Y. Dai, ChemCatChem, 2014, 6, 2456-2476.

37 S. Sarina, E. R. Waclawik and H. Zhu, Green Chem., 2013, 15, 1814-1833.

38 X. Zhou, G. Liu, J. Yu and W. Fan, J. Mater. Chem., 2012, 22, 21337-21354.

39 M. Zhu, P. Chen and M. Liu, J. Mater. Chem., 2011, 21, 16413-16419.

40 J. Jiang and L. Zhang, Chem.-Eur. J., 2011, 17, 3710-3717.

41 M. Zhu, P. Chen, W. Ma, B. Lei and M. Liu, ACS Appl. Mater. Interfaces, 2012, 4, 6386-6392.

42 M. Cui, J. Yu, H. Lin, Y. Wu, L. Zhao and Y. He, Appl. Surf. Sci., 2016, 387, 912-920.

43 C.-C. Shen, Q. Zhu, Z.-W. Zhao, T. Wen, X. Wang and A.-W. Xu, J. Mater. Chem. A, 2015, 3, 14661-14668.

44 L. Dong, Y. He, T. Li, J. Cai, W. Hu, S. Wang, H. Lin, M. Luo, X. Yi, L. Zhao, W. Weng and H. Wan, Appl. Catal., A, 2014, 472, 143-151. 
45 W.-S. Wang, H. Du, R.-X. Wang, T. Wen and A.-W. Xu, Nanoscale, 2013, 5, 3315-3321.

46 T. Li, Y. He, H. Lin, J. Cai, L. Dong, X. Wang, M. Luo, L. Zhao, X. Yi and W. Weng, Appl. Catal., B, 2013, 138-139, 95-103.

47 Q. Zhu, W.-S. Wang, L. Lin, G.-Q. Gao, H.-L. Guo, H. Du and A.-W. Xu, J. Phys. Chem. C, 2013, 117, 5894-5900.

48 S. Wang, S. Dong, J. Wang, L. Zhang, P. Han, C. Zhang, X. Wang, K. Zhang, Z. Lan and G. Cui, J. Mater. Chem., 2012, 22, 21051-21056.

49 X. D. Ren, R. Liu, L. M. Zheng, Y. P. Ren, Z. Z. Hu and H. He, Appl. Phys. Lett., 2016, 108, 071904.

50 H. Zhang, X. Lv, Y. Li, Y. Wang and J. Li, ACS Nano, 2010, 4, 380-386.
51 V. Causin, C. Marega, A. Marigo, G. Ferrara and A. Ferraro, Eur. Polym. J., 2006, 42, 3153-3161.

52 A. R. Tao, S. Habas and P. Yang, Small, 2008, 4, 310-325.

53 C. Chen, W. Ma and J. Zhao, Chem. Soc. Rev., 2010, 39, 42064219.

54 X. Zhang, M. Zhu, P. Chen, Y. Li, H. Liu, Y. Li and M. Liu, Phys. Chem. Chem. Phys., 2015, 17, 1217-1225.

55 Y. Wang, P. Chen, Y. Shen, C. Chen, C. Yang and M. Liu, Phys. Chem. Chem. Phys., 2015, 17, 25182-25190.

56 M. Adachi, M. Sakamoto, J. Jiu, Y. Ogata and S. Isoda, J. Phys. Chem. B, 2006, 110, 13872-13880.

57 C. Zhai, M. Zhu, F. Ren, Z. Yao, Y. Du and P. Yang, J. Hazard. Mater., 2013, 263, 291-298. 